\title{
Domínios da qualidade de vida associados à percepção de saúde: um estudo com idosas de um programa de atividade física em bairros de baixa renda de Curitiba, Paraná, Brasil
}

\author{
Aspects of quality of life associated with self-rated health: \\ a study of elderly women from a physical activity program \\ in low-income neighborhoods in Curitiba in the state of Paraná, \\ Brazil
}

\author{
Gislaine Cristina Vagetti ${ }^{1}$ \\ Natalia Boneti Moreira ${ }^{2}$ \\ Valter Cordeiro Barbosa Filho ${ }^{3}$ \\ Valdomiro de Oliveira ${ }^{2}$ \\ Camila Ferreira Cancian ${ }^{2}$ \\ Oldemar Mazzardo ${ }^{2}$ \\ Wagner de Campos ${ }^{2}$
}

${ }^{1}$ Colegiado de

Musicoterapia, Faculdade de Artes do Paraná. R. do

Funcionários, Cabral. 80.035-050 Curitiba PR Brasil.

gislainevagetti@hotmail.com

${ }^{2}$ Departamento de Educação

Física, Universidade Federal do Paraná.

${ }^{3}$ Departamento de Educação Física, Universidade Federal de Santa Catarina.

\begin{abstract}
This study sought to analyze which aspects of quality of life (QoL; Physical, Psychological, Social Relationships and Environmental) are associated with the perception of health among elderly women from low-income neighborhoods in Curitiba in the state of Paraná. The sample comprised 450 elderly participants of the Elderly in Motion Program of the Boqueirão, Pinheirinho and Bairro Novo neighborhoods. The question "How do you rate your general health?" was used to assess the perceived health in the elderly, and the Whoqol-Bref questionnaire was used to identify the aspects of QoL. Descriptive statistics, the Mann-Whitney U test, Spearman's correlation test and binary logistic regression were used for data analysis. All aspects were significantly associated with perceived health $(p<0.05)$. However, after adjusting for confounding variables, only the Physical and Environmental aspects revealed significant associations. In other words, elderly women with a negative perception of these aspects were twice as likely to have a negative perception of health. The conclusion reached is that programs aimed at promoting health and QoL in low-income elderly women ought to concentrate their actions on the physical and environmental aspects of $Q o L$.
\end{abstract}

Key words Quality of life, Health of the elderly, Environment
Resumo O presente estudo teve como objetivo analisar quais dominios da qualidade de vida (QV; Físico, Psicológico, Relações Sociais e Meio Ambiente) estão associados à percepção de saúde de idosas em bairros de baixa renda de Curitiba, Paraná. A amostra foi composta por 450 idosas participantes do programa Idoso em Movimento dos bairros: Boqueirão, Pinheirinho e Bairro Novo. A questão "De modo geral você diria que sua saúde é?" foi utilizada para avaliar a percepção de saúde das idosas, e o questionário WhoqolBref foi utilizado para identificar os dominios da QV. Para análise dos dados foram utilizados a estatística descritiva, o teste U de Mann-Whitney, a correlação de Spearman e a regressão logística binária. Todos os domínios estiveram significativamente associados com a percepção de saúde ( $p$ $<0,05$ ). Contudo, após ajuste para variáveis de confusão, os dominios Físico e Meio Ambiente apresentaram as associações de maior magnitude, ou seja, idosas com percepção negativa desses domínios tiveram cerca de duas vezes mais chances de ter uma percepção de saúde negativa. Em conclusão, programas que visam à promoção de saúde e $Q V$ em idosas de baixa renda devem nortear suas ações nos aspectos físicos e ambientais da qualidade de vida.

Palavras-chave Qualidade de vida, Saúde do idoso, Meio ambiente 


\section{Introdução}

A população idosa vem aumentando significativamente no Brasil ${ }^{1,2}$. Segundo o Instituto Brasileiro de Geografia e Estatística ${ }^{3}$, a população de indivíduos com 65 anos ou mais, que correspondia a $4,8 \%$ da população brasileira em 1991 , chegou a 7,4\% em 2010. Além disso, a expectativa de vida do brasileiro também aumentou, atingindo em 2010 a média de 73,1 anos. De acordo com Organização Mundial de Saúde ${ }^{4,5}$, até 2025, o Brasil será o sexto país do mundo em número de idosos, representando $15 \%$ da população nacional. Este aumento da população idosa brasileira, assim como ocorre em outros países, é reflexo da queda da mortalidade e da fecundidade, aliados ao aumento da longevidade, principalmente entre as mulheres ${ }^{1,6,7}$.

Entretanto, o aumento da expectativa de vida pode não significar uma melhora na qualidade de vida $(\mathrm{QV})$, que é definida pela OMS como "a percepção do individuo quanto à sua posição na vida, no contexto da cultura e do sistema de valores em que vive, levando em conta suas metas, expectativas, padrões e preocupações" ". Este é um conceito que incorpora a saúde física de uma pessoa, seu estado psicológico, seu nível de dependência, suas relações sociais, suas crenças e sua relação com características relevantes do ambiente ${ }^{9,10}$. Estudos de base populacional da população brasileira ${ }^{2}$ evidenciam que o processo de envelhecimento em si não está associado ao aumento das despesas médicas, mas o que encarece os gastos são as deficiências e a saúde precária, frequentemente associadas à velhice. Porém, se as pessoas envelhecerem com uma saúde melhor, as despesas médicas, provavelmente, não aumentarão de modo tão rápido, o que permitirá um menor comprometimento de aspectos físicos, sociais, psicológicos e cognitivos, que fazem parte do processo de envelhecimento e geram possíveis impactos na $\mathrm{QV}$ e na percepção de saúde do indivíduo ${ }^{11}$.

Diante do envelhecimento da população e sua busca por uma vida mais longa e com melhor QV, é crescente o interesse da comunidade científica brasileira e internacional em estudar a dos indivíduos nessa faixa etária e os fatores correlatos à percepção positiva de saúde, visto que o aumento do número de anos de vida deveria ser acompanhado pela melhoria ou manutenção das condições de saúde e da QV ${ }^{4,10,12,13}$.

Neste contexto, surgem as divergências sobre a interpretação da QV, percepção de saúde e dos fatores relacionados a estas variáveis, como os físicos e emocionais. Dessa maneira, com a intenção de facilitar essa interpretação foi criado o modelo hierárquico de Spilker ${ }^{13}$. Este modelo sugere que a QV deve ser analisada e interpretada a partir de três níveis, partindo do mais geral ao mais específico. O primeiro nível trata da QV global individual (incluindo percepção de saúde e satisfação com a vida); o segundo nível, avalia separadamente cada domínio, agrupando fatores específicos que influenciam na QV individual; por fim, no terceiro nível, encontram-se os fatores específicos que contribuem para a formação de cada domínio, ou seja, as facetas que compõem cada um, como por exemplo, o físico que é composto por: dor e desconforto, energia e fadiga, sono e repouso, atividades da vida cotidiana, dependência de medicação e tratamentos, mobilidade e capacidade de trabalho. Portanto, os autores estimulam o estudo dos aspectos de níveis inferiores que podem predizer a percepção de saúde e a QV do indivíduo ${ }^{13}$.

Alguns estudos testaram o modelo proposto por Spilker ${ }^{13} \mathrm{em}$ idosos. Arnold et al..$^{14}$ verificaram que os domínios psicológicos, sociais e físicos foram os melhores preditores da percepção de QV global e que a condição de saúde é um importante moderador desta associação ${ }^{14}$. Da mesma forma, uma pesquisa de Pereira et al. ${ }^{15}$ realizada em uma cidade de pequeno porte em Minas Gerais, destacou o domínio físico, seguido pelo ambiental e pelo psicológico, como principais preditores da QV global. Por fim, Vagetti et al. ${ }^{16} \mathrm{em}$ uma pesquisa realizada na cidade de Curitiba (PR) verificaram que aspectos físicos e de participação social são os mais importantes preditores da QV global (incluindo percepção de saúde e satisfação com a vida).

Esses estudos contribuem para a compreensão do modelo teórico de Spilker ${ }^{14}$ em idosos da população em geral, com diferentes problemas de saúde e fisicamente ativos. Contudo, ao nosso conhecimento, não foram realizados estudos testando esse modelo teórico especificamente em idosos de regiões pobres. Uma pior condição econômica (por exemplo, falta de dinheiro para compra de remédios e alimentos, dentre outras condições) pode ter impacto negativo na QV e na percepção de saúde em idosos ${ }^{17-19}$. Neste contexto, a OMS revela que a maior parte da carga de doenças, assim como as iniquidades em saúde, que existem em todos os países, acontece por conta das condições em que as pessoas nascem, vivem, crescem, trabalham e envelhecem. Esse conjunto de condições é chamado "determinantes sociais da saúde", um termo que resume os 
determinantes sociais, econômicos, políticos, culturais e ambientais da saúde, como por exemplo, a distribuição de renda do indivíduo que estratifica a sociedade e influencia diretamente na posição ocupada pelos indivíduos, causando iniquidades em saúde mais profundas, ou seja, as discrepâncias atribuíveis a esses mecanismos moldam a saúde dos indivíduos por meio de determinantes intermediários, como as condições de moradia e psicossociais, fatores comportamentais e biológico ${ }^{20}$. Portanto, investigações nesta direção poderão contribuir para entender quais domínios da QV (baseando-se nos domínios Físico, Psicológico, Relações Sociais e Meio Ambiente) contribuem para uma percepção negativa de saúde nessa população de risco. Tal população é assim classificada por apresentar uma maior exposição e vulnerabilidade a condições de risco à saúde, como uma má alimentação e habitação inadequada. Desta maneira, na aplicação prática em programas de promoção da QV e saúde no idoso, essa análise indicará os aspectos que podem nortear o desenvolvimento das ações de intervenção (por exemplo, promoção de um meio ambiente ou de relações sociais mais saudáveis) voltada a uma melhor percepção de saúde na população idosa de regiões de baixa renda.

Baseando-se nessas premissas, o objetivo do presente estudo foi investigar quais domínios da QV (Físico, Psicológico, Relações Sociais e Meio Ambiente) estão associados com a percepção de saúde em idosas participantes de um programa de atividade física em bairros de baixa renda de Curitiba (PR), Brasil.

\section{Materiais e métodos}

\section{Delineamento do estudo e amostra}

A presente pesquisa faz parte do projeto intitulado "Relação entre nível de atividade física, capacidade funcional e qualidade de vida em idosas", realizado pelo Centro de Pesquisa em Exercício e Esporte da Universidade Federal do Paraná. Seus integrantes previamente treinados entrevistaram 1806 idosas participantes do programa Idoso em Movimento, desenvolvido pela Secretaria Municipal de Esporte e Lazer. Foram selecionadas para o estudo apenas idosas, devido ao número muito reduzido de homens participantes do programa.

Para compor a amostra do presente estudo de caráter transversal foram selecionadas de maneira intencional 450 idosas residentes nos três bairros classificados como de baixa renda, de acordo com as estimativas do Instituto de Pesquisa e Planejamento Urbano de Curitiba (PR ${ }^{21}$, tendo por base a renda média dos responsáveis pelos domicílios particulares da cidade, tais informações estão sumarizadas na Tabela 1. Das nove regiões administrativas de Curitiba (PR), a renda média do responsável familiar era inferior a 1.000 reais em apenas três regiões: Boqueirão, Pinheirinho e Bairro Novo. Portanto, as idosas usuárias dos centros de atendimento dessas regiões administrativas, avaliadas no levantamento principal, foram selecionadas para a amostra final do presente estudo.

\section{Instrumentos e procedimentos}

A coleta de dados foi realizada de fevereiro a julho de 2011. Os dados foram obtidos por meio de entrevistas estruturadas individualizadas com questionários, aplicados por avaliadores previamente treinados do Centro de Pesquisa em Exercício e Esporte da Universidade Federal do Paraná.

A QV das idosas foi avaliada mediante a utilização do Whoqol-Bref, um instrumento com propriedades psicométricas satisfatórias na investigação da $\mathrm{QV}$ em idosos brasileiros ${ }^{22,23}$. Este instrumento possui 26 itens, dois relacionados à QV global e saúde em geral, e os 24 demais sendo categorizados em quatro domínios (Físico, Meio Ambiente, Relações Sociais e Psicológico ${ }^{24}$. Os domínios são compostos por questões, cujas pontuações das respostas variam entre 1 e 5 . A partir disso, os escores finais de cada domínio foram calculados por uma sintaxe, com base nos critérios propostos pelo grupo $\mathrm{WHOQOL}^{8}$, que classifica os domínios da QV, com escores percentuais que variam de 0 a 100 . Quanto mais próximo de 100, melhor é a QV do avaliado.

Um escore geral de QV também foi realizado por meio da média dos escores nos 4 domínios (Escore médio dos 4 domínios). Adicionalmente, para fins de análise, os escores dos domínios foram classificados dicotomicamente (positivo ou negativo), considerando a mediana de cada domínio. Portanto, a idosa com o escore de um determinado domínio inferior à mediana foi classificada com percepção negativa deste; caso o escore tenha sido igual ou maior à mediana, a idosa foi classificada com percepção positiva. As medianas obtidas foram 71,42, 75,00, 66,67, 68,00 e 69,75 para os domínios Físico, Psicológico, Relações Sociais, Meio Ambiente e Escore médio dos 4 domínios, respectivamente. 
Tabela 1. Valor do rendimento nominal médio e mediano mensal das pessoas com rendimento, responsáveis pelos domicílios particulares permanentes, segundo a região administrativa de Curitiba ${ }^{22}$.

\begin{tabular}{|c|c|c|c|c|}
\hline \multirow{3}{*}{ Região Administrativa } & \multicolumn{4}{|c|}{$\begin{array}{c}\text { Rendimento mensal das pessoas com rendimento, responsáveis } \\
\text { pelos domicílios particulares permanentes }\end{array}$} \\
\hline & \multicolumn{2}{|c|}{ Em R\$ } & \multicolumn{2}{|c|}{ Em salários mínimos * } \\
\hline & Médio & Mediano & Médio & Mediano \\
\hline Matriz & $2.904,79$ & $2.000,00$ & 19,24 & 13,25 \\
\hline Boqueirão $^{* *}$ & 970,24 & 600,00 & 6,43 & 3,97 \\
\hline Cajuru & $1.050,74$ & 600,00 & 6,96 & 3,97 \\
\hline Boa Vista & $1.268,00$ & 700,00 & 8,40 & 4,64 \\
\hline Santa Felicidade & $1.364,44$ & 650,00 & 9,04 & 4,30 \\
\hline Portão & $1.563,26$ & 800,00 & 10,35 & 5,30 \\
\hline Pinheirinho ${ }^{* *}$ & 711,41 & 490,00 & 4,71 & 3,25 \\
\hline Bairro Novo ${ }^{* *}$ & 601,71 & 450,00 & 3,98 & 2,98 \\
\hline Curitiba & $1.430,96$ & 700,00 & 9,48 & 4,64 \\
\hline
\end{tabular}

Fonte: Adaptado de IPPUC - Banco de Dados 2000 (Características da População e dos Domicílios - Resultados do Universo) ${ }^{22}$. Salário mínimo utilizado: $\mathrm{R} \$ 151,00 .{ }^{* *}$ Regiões administrativas de Curitiba-PR consideradas de baixa renda média (menos que 1.000 reais). Idosas participantes dos centros de convivência desses bairros foram consideradas para o presente estudo.

A variável dependente deste estudo foi a percepção de saúde, avaliada por meio da seguinte questão: "De modo geral você diria que sua saúde é?". A idosa podia classificar sua saúde em uma escala Likert com pontos 1 (muito ruim), 2 (ruim), 3 (nem boa, nem ruim), 4 (boa) e 5 (excelente). Idosas que reportaram os pontos 1 e 2 foram classificadas com uma percepção de saúde negativa.

Algumas variáveis foram analisadas para descrição da amostra e possíveis variáveis de confusão da associação entre os domínios da QV e a percepção de saúde nas idosas. A classe econômica foi avaliada por meio do questionário da Associação Brasileira de Empresas de Pesquisa ${ }^{25}$. Este critério visa estimar o poder de compra das pessoas e famílias e o grau de instrução do chefe da família, separando nas classes econômicas de A (mais alta) a E (mais baixa). As classes econômicas foram agrupadas em classe alta $(A+B)$, classe média $(\mathrm{C})$ e classe baixa $(\mathrm{D}+\mathrm{E})$. Além disso, outras variáveis sociodemográficas foram coletadas: idade, estado civil (solteira, casada, separada e viúva), situação ocupacional (aposentada, pensionista, dona de casa e outra) e anos de escolaridade (posteriormente classificados como analfabeto, primário completo, fundamental completo, superior completo e pós-graduação).

Por fim, o estado nutricional e o nível de atividade física também foram estudados como possíveis variáveis de confusão. O peso corporal $(\mathrm{kg})$ e a estatura $(\mathrm{m})$ foram mensurados para cálculo do Índice de Massa Corporal (IMC, kg/ $\mathrm{m}^{2}$ ). O IMC foi classificado de acordo com a proposta da $\mathrm{OMS}^{26}$ : baixo peso $\left(\leq 18,5 \mathrm{~kg} / \mathrm{m}^{2}\right)$, peso normal $\left(18,6-24,9 \mathrm{~kg} / \mathrm{m}^{2}\right)$, sobrepeso $(25-$ $\left.29,9 \mathrm{~kg} / \mathrm{m}^{2}\right)$ e obesidade $\left(\geq 30 \mathrm{~kg} / \mathrm{m}^{2}\right)$.

O tempo semanal (semana prévia à coleta de dados) que a idosa participou em atividades físicas foi estimado por meio da versão curta do International Physical Acitivy Questionnaire (IPAQ), validado para a população brasileira ${ }^{27}$. As idosas que realizaram ao menos 150 minutos de atividade física na semana anterior à pesquisa foram consideradas como suficientemente ativas, enquanto as demais foram consideradas como insuficientemente ativas ${ }^{28}$.

\section{Aspectos éticos}

O Comitê de Ética em Pesquisa do Setor de Ciências da Saúde da Universidade Federal do Paraná aprovou o presente estudo. Todas as idosas que participaram deste assinaram um termo de consentimento livre e esclarecido.

\section{Tratamento estatístico}

A análise descritiva foi utilizada para caracterização das variáveis do estudo, baseando-se na frequência absoluta e relativa para as variáveis categóricas, e na média, mediana, valor mínimo 
e valor máximo para as contínuas. O teste de Kolmogorov-Smirnov foi aplicado para identificar a normalidade dos escores de percepção de saúde e dos domínios da QV. Optou-se por utilizar estatísticas inferenciais não paramétricas, uma vez que os dados não apresentaram distribuição normal. A correlação entre os escores de percepção de saúde e os domínios da QV foi avaliada mediante o teste de Spearman. O teste de Mann-Whitney foi utilizado para identificar diferenças nos escores de cada domínio da QV entre as categorias da percepção de saúde (positiva e negativa). A associação bivariada entre a percepção de saúde e cada domínio da QV, classificados dicotomicamente (positiva ou negativa), foi realizada por meio do teste de Qui-quadrado. Adicionalmente, modelos de regressão logística binária bruta e ajustada foram construídos para identificar a associação entre as variáveis, mediante estimativas de odds ratio e seus respectivos intervalos de confiança de 95\% (IC 95\%). Nesta análise, a percepção de saúde foi a variável dependente (considerando a percepção de saúde negativa como desfecho de interesse) e os domínios da QV foram inseridos como variáveis independentes. Os modelos de regressão foram ajustados para idade, tempo semanal de atividade física e anos de escolaridade como variáveis contínuas, e classificação do IMC, classe econômica e situação ocupacional como variáveis categóricas. Todas as análises foram realizadas no programa SPSS 18.0, adotando-se o nível de significância de 5\%.

\section{Resultados}

Conforme se verifica na Tabela 2, a amostra apresentou uma maior proporção de idosas com idades de 60 a 69 anos $(65,6 \%)$, casadas ou que possuem um companheiro $(47,7 \%)$, aposentadas $(52,1 \%)$, com ensino fundamental incompleto (71\%), de classe econômica C (63,9\%), suficientemente ativas ( $\geq 150$ minutos/semana) $(85,1 \%)$ e com sobrepeso $(45,7 \%)$.

A Tabela 3 mostra os valores descritivos da percepção de saúde, segundo a classificação de cada domínio da QV em positivo ou negativo. Os escores da percepção de saúde foram estatisticamente maiores entre as idosas com percepção positiva dos domínios da QV, em comparação aos pares com percepção negativa (todos com $\mathrm{p}<0,001)$. Essas diferenças estatísticas foram obtidas em todos os domínios da QV, contudo, as maiores diferenças nos escores médios
Tabela 2. Descrição da amostra do estudo $(\mathrm{n}=450)$.

\begin{tabular}{|c|c|c|}
\hline Variáveis & $\mathbf{n}$ & $\%$ \\
\hline \multicolumn{3}{|l|}{ Faixa etária } \\
\hline $60-69$ & 295 & 65,6 \\
\hline $70-79$ & 130 & 28,9 \\
\hline Acima de 80 & 25 & 5,5 \\
\hline \multicolumn{3}{|l|}{ Estado civil } \\
\hline Solteira (nunca casou) & 27 & 6,0 \\
\hline Casada ou com companheiro & 214 & 47,7 \\
\hline Separada/Divorciada & 39 & 8,6 \\
\hline Viúva & 170 & 37,7 \\
\hline \multicolumn{3}{|l|}{ Situação Ocupacional } \\
\hline Aposentada & 234 & 52,1 \\
\hline Pensionista & 86 & 19,2 \\
\hline Dona de Casa & 125 & 27,8 \\
\hline Outro & 4 & 0,9 \\
\hline \multicolumn{3}{|l|}{ Escolaridade da idosa } \\
\hline Analfabeto & 18 & 4,0 \\
\hline Fundamental incompleto & 319 & 71,0 \\
\hline Superior incompleto & 100 & 22,3 \\
\hline Superior completo & 9 & 1,8 \\
\hline Pós-Graduação & 4 & 0,9 \\
\hline \multicolumn{3}{|l|}{ Classe econômica } \\
\hline A+B (melhor condição) & 107 & 23,9 \\
\hline $\mathrm{C}$ & 287 & 63,7 \\
\hline D+E (pior condição) & 56 & 12,4 \\
\hline \multicolumn{3}{|c|}{$\begin{array}{l}\text { Nível de atividade física (150 minutos/ } \\
\text { por semana) }\end{array}$} \\
\hline Suficientemente ativa & 383 & 85,1 \\
\hline Insuficientemente ativa & 67 & 14,9 \\
\hline \multicolumn{3}{|l|}{ Classificação do IMC } \\
\hline Desnutrição & 1 & 2,0 \\
\hline Peso normal & 98 & 21,7 \\
\hline Sobrepeso & 205 & 45,7 \\
\hline Obesidade & 146 & 32,4 \\
\hline
\end{tabular}

de percepção de saúde foram encontradas no domínio Físico (3,22 vs. 2,70) e no Escore geral dos 4 domínios (3,20 vs. 2,70).

Os valores de correlação de Spearman obtidos entre a percepção de saúde e os escores de cada domínio da QV estão descritos na Tabela 4. Todos estes domínios estiveram significativamente associados à $\mathrm{QV}$ (todos com $\mathrm{p}<0,001)$. Aquele com menor correlação com a percepção de saúde foi Relações Sociais, enquanto que as maiores foram encontradas para o Psicológico, o Físico e o Escore médio dos 4 domínios.

$\mathrm{Na}$ Tabela 5 podem ser observados os indicadores de associação entre a percepção de saúde e os domínios da QV. Oito em cada dez idosas participantes deste estudo apresentavam uma percepção de saúde negativa. $\mathrm{Na}$ análise bivaria- 


\begin{tabular}{|c|c|c|c|c|c|c|c|c|}
\hline \multirow[t]{8}{*}{ 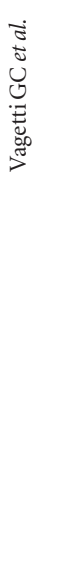 } & $\begin{array}{l}\text { Tabela 3. Média, mediana e val } \\
\text { domínios da QV em negativa e }\end{array}$ & $\begin{array}{l}\text { or mínim } \\
\text { positiva }\end{array}$ & $\begin{array}{l}\text { o e máximo } \\
\text { em idosas }(1\end{array}$ & $\begin{array}{l}\text { da percepção } \\
=450) .\end{array}$ & le saúde d & acordo co & $\mathrm{m}$ a classifica & ão dos \\
\hline & \multirow{2}{*}{ Domínios da QV } & \multicolumn{3}{|c|}{ Percepção negativa do domínio } & \multicolumn{3}{|c|}{ Percepção positiva do domínio } & \multirow{2}{*}{$\mathbf{P}^{*}$} \\
\hline & & Média & Mediana & Mín-Máx & Média & Mediana & Mín-Máx & \\
\hline & Físico & 2,70 & 3,00 & $1-4$ & 3,22 & 3,00 & $2-4$ & $<0,001$ \\
\hline & Psicológico & 2,81 & 3,00 & $1-4$ & 3,24 & 3,00 & $2-4$ & $<0,001$ \\
\hline & Relações Sociais & 2,77 & 3,00 & $1-4$ & 3,16 & 3,00 & $2-4$ & $<0,001$ \\
\hline & Meio Ambiente & 2,74 & 3,00 & $1-4$ & 3,21 & 3,00 & $2-4$ & $<0,001$ \\
\hline & Escore médio dos 4 domínios & 2,70 & 3,00 & $1-4$ & 3,20 & 3,00 & $2-4$ & $<0,001$ \\
\hline
\end{tabular}

*Valores obtidos a partir do teste U de Mann-Whitney. QV = qualidade de vida; Mín-Máx = valor mínimo e máximo.

Tabela 4. Correlação de Spearman entre percepção de saúde e os escores dos domínios da QV em idosas $(\mathrm{n}=450)$.

\begin{tabular}{lcc}
\hline \multicolumn{1}{c}{ Domínios da QV } & $\begin{array}{c}\text { Coeficiente de } \\
\text { Spearman }(\rho)\end{array}$ & $\mathrm{P}^{*}$ \\
\hline Físico & 0,533 & $<0,001$ \\
Psicológico & 0,503 & $<0,001$ \\
Relações Sociais & 0,362 & $<0,001$ \\
Meio Ambiente & 0,457 & $<0,001$ \\
Escore médio dos 4 domínios & 0,561 & $<0,001$ \\
\hline
\end{tabular}

"Valores a partir do teste da correlação de Spearman. da utilizando o teste de Qui-quadrado, verificou-se uma associação direta entre os domínios da QV e a percepção de saúde. Foram encontradas maiores porcentagens de percepção de saúde negativa entre as idosas que também apresentavam uma percepção negativa do domínio da $\mathrm{QV}$ (com base na mediana), independentemente do domínio avaliado $(\mathrm{p}<0,05)$.

$\mathrm{Na}$ análise do odds ratio bruto, observou-se uma forte associação da percepção de saúde com o Escore médio dos 4 domínios ( $\mathrm{OR}=3,69$, IC95\%: 2,13-6,39), sendo que o domínio da QV

Tabela 5. Porcentagem de idosas com percepção de saúde negativa de acordo com as categorias de cada domínio da QV (positiva e negativa), e valores de odds ratio brutos e ajustados entre a percepção de saúde e os domínios da QV $(\mathrm{n}=450)$.

\begin{tabular}{|c|c|c|c|c|}
\hline \multirow{2}{*}{ Domínios da QV } & \multirow[b]{2}{*}{$\mathbf{n}$} & \multicolumn{3}{|c|}{ Percepção de Saúde Negativa } \\
\hline & & $\%$ & $\begin{array}{r}\text { OR bruto } \\
\text { (IC 95\%) }\end{array}$ & $\begin{array}{l}\text { OR ajustado } \\
(\text { IC } 95 \%)^{* *}\end{array}$ \\
\hline Total & 450 & $373(82,9)$ & & \\
\hline \multicolumn{5}{|l|}{ Físico } \\
\hline Negativa $(<71,42)$ & 233 & $213(91,4)^{*}$ & $3,86(2,23-6,68)$ & $2,02(1,03-3,95)$ \\
\hline Positiva $(\geq 71,42)$ & 217 & $160(73,4)$ & 1 & 1 \\
\hline \multicolumn{5}{|l|}{ Psicológico } \\
\hline Negativa $(<75,00)$ & 308 & $274(89,0)^{*}$ & $3,58(2,17-5,92)$ & $1,99(1,06-3,74)$ \\
\hline Positiva $(\geq 75,00)$ & 142 & $99(69,2)$ & 1 & 1 \\
\hline \multicolumn{5}{|l|}{ Relações Sociais } \\
\hline Negativa $(<66,67)$ & 250 & $223(89,2)^{*}$ & $2,80(1,69-4,68)$ & $1,78(1,01-3,14)$ \\
\hline Positiva $(\geq 66,67)$ & 200 & $150(74,6)$ & 1 & 1 \\
\hline \multicolumn{5}{|l|}{ Meio Ambiente } \\
\hline Negativa $(<68,00)$ & 251 & $227(90,4)^{*}$ & $3,50(2,07-5,90)$ & $2,46(1,21-4,97)$ \\
\hline Positiva $(\geq 68,00)$ & 199 & $146(73,0)$ & 1 & 1 \\
\hline \multicolumn{5}{|l|}{ Escore médio dos 4 domínios } \\
\hline Negativa $(<69,75)$ & 229 & $209(91,3)^{*}$ & $3,69(2,13-6,39)$ & $0,73(0,30-1,78)$ \\
\hline Positiva $(\geq 69,75)$ & 221 & $164(73,9)$ & 1 & 1 \\
\hline
\end{tabular}

* Diferenças significativas de acordo com o teste de Qui-quadrado, com $\mathrm{p}<0,01$. ${ }^{* *}$ Ajustado para idade, tempo semanal de atividade física, anos de escolaridade, classificação do IMC, classe econômica, situação ocupacional e as demais variáveis independentes inseridas no modelo. IC: intervalo de confiança de $95 \%$; OR: odds ratio 
mais fortemente associado foi o Físico. As idosas com percepção negativa do domínio Físico (escores inferiores à mediana) tiveram aproximadamente quatro vezes mais chances $(\mathrm{OR}=3,86$, IC95\%: 2,23-6,68) de ter uma percepção de saúde negativa, em comparação com seus pares com percepção positiva do domínio Físico. Os demais domínios também estiveram diretamente associados com a percepção de saúde nas idosas, com valores de odds ratio variando de 2,80 (Relações Sociais) a 3,58 (Psicológico).

$\mathrm{Na}$ análise ajustada para variáveis de confusão (idade, tempo semanal de atividade física, escolaridade, classe econômica, situação ocupacional e classificação do IMC), somente o Escore geral dos 4 domínios perdeu associação significativa com a percepção de saúde $(\mathrm{OR}=0,73$, IC95\%: 0,30-1,78). Os demais domínios da QV estiveram significativa e independentemente associados com a percepção de saúde. Contudo, os domínios da QV com maiores associações foram o Meio Ambiente, Físico e Psicológico. Após ajuste para variáveis de confusão, as idosas que tinham uma percepção negativa do domínio Meio Ambiente tiveram 2,5 vezes mais chances de reportar uma percepção de saúde negativa, em comparação aos seus pares com percepção positiva desse domínio. De modo semelhante, as idosas com percepção negativa do domínio Físico e Psicológico tiveram 2 vezes mais chances de ter uma percepção de saúde negativa, em comparação aos seus pares com percepção positiva desse domínio.

\section{Discussão}

A QV é composta por vários aspectos, entre eles a percepção da saúde, que pode gerar uma visão global da saúde e do bem-estar de uma perspectiva individual ${ }^{29}$. Com o avanço da idade há um aumento de condições crônicas e alterações funcionais que resultam em comprometimentos físicos, emocionais e/ou psicológicos; consequentemente, o avanço da idade pode reduzir a percepção de saúde e a $\mathrm{QV}^{30}$. A percepção de saúde tem merecido uma atenção especial, pois tem sido descrita como um indicador associado ao declínio da autonomia funcional e até mesmo da mortalidade entre idosos, apresentando o potencial de sintetizar uma complexa interação de fatores envolvidos em sua saúde ${ }^{31}$. Portanto, é fundamental o estudo de variáveis que podem estar associadas a uma percepção de saúde negativa entre idosas, contribuindo para a efetividade de ações de intervenção que busquem promover a saúde e a QV nessa população.

O presente estudo revelou uma elevada prevalência $(82,9 \%)$ de percepção de saúde negativa em idosas que residiam em bairros de baixa renda. Tal fato é evidenciado pela condição socioeconômica das idosas, que revelou um elevado percentual na classe econômica C ou D+E (76,1\%) e baixa escolaridade $(75,0 \%)$, o que reforça que grande parte das idosas avaliadas estavam expostas a condições socioeconômicas precárias. Corroborando com esse achado, Lima-Costa et al. ${ }^{19}$ analisaram os dados da Pesquisa Nacional de Amostra por Domicílios de 1998, realizada com cerca de 20 mil idosos brasileiros, e evidenciaram que aqueles com menor renda domiciliar apresentam uma pior percepção de saúde em comparação aos de melhor condição financeira. $\mathrm{Ng}$ et al. ${ }^{17}$ avaliaram idosos de uma zona rural da Indonésia e também encontraram uma forte e direta associação entre o nível socioeconômico e a percepção da saúde. Essas evidências também foram encontradas em outras pesquisas com idosos brasileiros ${ }^{18,32,33}$, e de outros países ${ }^{34,35}$.

Diante dessas evidências, nota-se que piores condições socioeconômicas como má qualidade da habitação, dependência econômica ou instabilidade financeira, contribuem diretamente para uma percepção de saúde negativa entre idosos. No presente estudo as idosas avaliadas eram participantes de um programa de atividade física e, embora esta esteja associada a uma melhor $\mathrm{QV}^{15}$, grande parte delas apresentou uma percepção de saúde negativa. Com isso, as políticas públicas de promoção da saúde em idosos devem buscar ferramentas adicionais que contribuam para uma melhor QV e saúde no subgrupo de baixa renda. Essas ações de intervenção, além de estimular a prática de atividade física e relacionamentos sociais, devem promover um ambiente com melhores condições estruturais (por exemplo, acesso a alimentação saudável, boas condições de higiene e atividades educacionais) dentro e fora do centro de atividades. Para que essas ações possam ser concretizadas, é importante a criação de novos programas com enfoque multidisciplinar, para assim abranger vários aspectos de saúde do indivíduo, proporcionando uma melhor condição de vida e possivelmente melhores QV e saúde. Com isso, o desenvolvimento de ações específicas para esse subgrupo de risco pode contribuir para a promoção de saúde e QV na população idosa brasileira como um todo.

Ao analisar quais aspectos da QV estão associados à percepção de saúde em idosas de bair- 
ros de baixa renda, verificou-se que os 4 domínios (Físico, Psicológico, Relações Sociais e Meio Ambiente) estão significativa e independentemente associados com a percepção de saúde. Contudo, após ajustes para variáveis de confusão, os domínios Físico, Psicológico e Meio Ambiente tiveram a associação de maior magnitude com a percepção de saúde nas idosas avaliadas (Tabela 5). Esses resultados sugerem que os aspectos físicos (percepção de dor, desconforto e fadiga, energia para o dia-a-dia e condições de sono e repouso), psicológicos (sentimentos positivos e negativos, pensar, aprender, memória e concentração, autoestima, imagem corporal e aparência, espiritualidade, religião e crenças pessoais), e relacionados ao ambiente (segurança física, ambiente no lar, recursos financeiros, cuidados de saúde e sociais, oportunidades de lazer e ambiente físico), que compõem os domínios Físico, Psicológico e Meio Ambiente da QV, são importantes preditores da percepção de saúde geral entre idosas de bairros de baixa renda.

Os resultados do presente estudo apresentam algumas semelhanças, mas também diferenças, com outras pesquisas que testaram o modelo teórico de Spilker ${ }^{14}$ em outros grupos de idosos. Um estudo com idosas ativas ${ }^{15}$ também destacou o domínio Físico como um importante preditor da QV global (incluindo percepção de saúde e satisfação com a vida) nesse grupo. Contudo, a participação social (aspectos sociais específicos da população idosa) foi o outro aspecto preditor da QV global nas idosas ativas ${ }^{15}$. Já no estudo de Pereira et al. ${ }^{15}$, com idosas de uma cidade de pequeno porte (Teixeira, MG), os domínios que melhor predisseram a QV global foram o Físico e o Meio Ambiente, como observado no presente estudo. Por fim, Arnold et al. ${ }^{14}$ realizaram um estudo com idosos de diferentes condições de saúde (indivíduos saudáveis e com condições como hipertensão, diabetes, problemas osteomusculares, entre outros) e destacaram o domínio Psicológico como preditor da QV global e percepção de saúde. Desta forma, percebe-se que o nível de atividade física e a condição de saúde são importantes moderadores da associação entre percepção de saúde e os aspectos específicos da QV.

O presente estudo acrescenta à literatura o conceito de que, em idosas que vivem em regiões de baixa renda, aspectos Físicos, Psicológicos e do Meio Ambiente se destacam na predição da percepção de saúde nesse subgrupo de risco. Portanto, esses resultados sugerem que a situação socioeconômica também pode ser um importante moderador no estudo do modelo teórico de Spi-
$1 \mathrm{ker}^{13}$. Estudos futuros são necessários para testar essa hipótese, estratificando seus resultados em idosos de diferentes condições socioeconômicas.

Devido à forte associação do domínio Físico e a percepção de saúde em idosas de bairros de baixa renda, o desenvolvimento de aspectos físicos, mediante a promoção de atividade física, desenvolvimento da aptidão física e estímulo para o estilo de vida ativo fora do centro de atendimento, pode ser uma ferramenta importante para a promoção de uma percepção de saúde positiva entre as idosas. De fato, a atividade física é de grande importância para a manutenção da saúde para os idosos ${ }^{36}$. A atividade física regular, além de aumentar a expectativa de vida e reduzir o risco de doenças crônicas, contribui de maneira significativa para a manutenção da aptidão física e de capacidades funcionais como andar, tomar banho e comer ${ }^{37}$.

Da mesma maneira, outro fator importante para a promoção de uma percepção de saúde positiva entre as idosas é o domínio Psicológico, que se mostrou fortemente associado à percepção de saúde das idosas avaliadas. Deste modo, a participação em atividades no centro de atendimento é capaz de oferecer às idosas um trabalho com profissionais qualificados e interessados em ajudá-las em diferentes aspectos, desde saúde até relações interpessoais, o que contribui para a formação de novos círculos de convivência, para uma melhor autoestima e sentimentos positivos, podendo assim explicar a forte associação desse domínio com a percepção de saúde em idosas de bairros de baixa renda, o que é confirmado por outros estudos $^{13,38,39}$, indicando sua importância frente à promoção de $\mathrm{QV}$ de idosas.

Além disso, outra dimensão que deve ser considerada nas políticas de promoção da saúde do idoso é o meio ambiente. Segundo a $\mathrm{OMS}^{40}$, o ambiente em que o idoso está inserido pode determinar a dependência ou não do individuo. Dessa forma, é mais provável que um idoso esteja física e socialmente ativo se puder ir andando com segurança à casa de seus vizinhos, ao parque ou utilizar o transporte local. Idosos que vivem em ambientes inseguros são menos propensos a saírem sozinhos e, portanto, estão mais susceptíveis ao isolamento e à depressão, bem como a ter mais problemas de mobilidade e pior estado físico, o que vem a influenciar a QV e a sua percepção de saúde. Aspectos de incentivo quanto ao domínio ambiental, como promover acesso a locais para a atividade física e reduzir a insegurança pública, podem contribuir para a promoção de percepção de saúde em idosas. 
Essas evidências têm sido confirmadas por outros estudos ${ }^{41,42}$ indicando a importância de aspectos ambientais, sobretudo os relacionados à situação econômica (por exemplo, qualidade do ambiente em que reside, segurança e acesso a cuidados de saúde e lazer), que estão associados à percepção de saúde e QV em idosos.

Este estudo, que propõe uma avaliação do modelo teórico de Spilker ${ }^{13}$, incluindo os domínios da QV que estão mais fortemente associados com a percepção de saúde em idosas de bairros de baixa renda em Curitiba (PR), apresenta algumas limitações. A primeira foi a seleção de idosas participantes em centros que ofertavam atividades físicas. Portanto, não devem ser realizadas extrapolações para a população do município total ou de todas as regiões de baixa renda do município. Adicionalmente, a amostra incluiu somente mulheres idosas, impossibilitando a extensão dos resultados para indivíduos do gênero masculino. Diante dessas limitações, os resultados do presente estudo devem ser interpretados com devida cautela.

\section{Conclusão}

Os resultados do presente estudo indicaram uma elevada proporção de idosas de bairros de baixa renda com uma percepção de saúde negativa. Oito em cada dez idosas autoavaliaram a sua saúde como ruim ou muito ruim. Este resultado sugere que as idosas de regiões em situações de pior condição socioeconômica devem ter atenção especial em políticas de promoção da saúde na população desta faixa etária.

Todos os domínios da QV estiveram significativamente associados com a percepção de saúde. Contudo, após ajustes para variáveis de confusão como tempo semanal de atividade física, estado nutricional e classe econômica, os domínios Físico, Psicológico e Meio Ambiente apresentaram as associações de maior magnitude com a percepção de saúde. Estes resultados sugerem que problemas em aspectos físicos (fadiga durante o dia-a-dia, problemas de sono, etc.), psicológicos (sentimentos positivos e negativos, autoestima, etc.) e ambientais (insegurança física na comunidade e no lar, baixos recursos financeiros, falta de oportunidades de lazer, etc.) são importantes preditores de uma percepção de saúde negativa e uma baixa QV global em idosas.

Diante desses resultados, sugere-se que tanto os programas de atividade física quanto outros de atendimento ao idoso de regiões de baixa renda, devem nortear suas ações para os domínios Físicos, Psicológicos e Ambientais da QV. O foco de ações nesses aspectos poderá contribuir para a promoção de saúde do idoso em subgrupos de risco, como aqueles que estão expostos a condições socioeconômicas precárias.

\section{Colaboradores}

GC Vagetti participou de todas as etapas do estudo, incluindo a coleta e análise dos dados, e redação do manuscrito. VC Barbosa Filho participou da análise dos dados e redação do manuscrito. NB Moreira participou da coleta de dados e da redação do manuscrito. V de Oliveira, CF Cancian, O Mazzardo e W de Campos participaram da revisão crítica do manuscrito. Todos os autores participaram da aprovação final da versão a ser publicada.

\section{Agradecimentos}

O presente estudo foi financiado pela Fundação Araucária de Apoio ao Desenvolvimento Científico e Tecnológico do Paraná (FAP) e Coordenação de Aperfeiçoamento de Pessoal de Nível Superior (CAPES), sendo este trabalho realizado com apoio da Universidade Federal do Paraná (UFPR). 


\section{Referências}

1. Virtuoso JF, Mazo GZ, Menezes EC, Cardoso AS, Dias RG, Balbé GP. Perfil de morbidade referida e padrão de acesso a serviços de saúde por idosos praticantes de atividade física. Cien Saude Colet 2012; 17(1):23-31.

2. Monteiro RBC, Laurentino GEC, Melo PG, Cabral DL, Correa JCF, Teixeira-Salmela LF. Medo de cair e sua relação com a medida da independência funcional e a qualidade de vida em indivíduos após Acidente Vascular Encefálico. Cien Saude Colet 2013; 18(7):2017-2027.

3. Instituto Brasileiro de Geografia e Estatística (IBGE). Cresce expectativa de vida entre brasileiros. Rio de Janeiro: IBGE; 2010.

4. Organização Mundial da Saúde (OMS). Envelhecimento ativo: uma política de saúde. Brasília: Organização Pan-Americana da Saúde (OPAS), OMS; 2005.

5. Gai J, Gomes L, Nóbrega OT, Rodrigues MP. Fatores associados a quedas em mulheres idosas residentes na comunidade. Rev Ass Med Bras 2010; 56(3):327-332.

6. Instituto Brasileiro de Geografia e Estatística (IBGE). População brasileira envelhece em ritmo acelerado. Rio de Janeiro: IBGE; 2008.

7. Garrido GE, Furuie SS, Buchpiguel CA, Bottino CMC, Almeida OP, Cid CE. Relation between medial temporal atrophy and functional brain activity during memory processing in Alzheimer's disease: a combined MRI and SPECT study. J Neurol Neurosurg Psychiatry 2002; 73(5):508-516.

8. Whoqol. SRPB Group. A cross-cultural study of spirituality, religion and personal beliefs as components of quality of life. Soc Sci Med 2006; 62(6):1486-1497.

9. Mazo GZ, Mota JAPS, Cardoso AS, Prado APM, Antunes G. Qualidade de vida de mulheres idosas: comparação entre o nível de atividade física. Rev Arquivos em Mov 2009; 5(1):30-44.

10. Rufine MFC, Formiga NS, Valentini F, Melo GF. Escala de qualidade de vida: análise estrutural de uma versão para idosas. Cien Saude Colet 2013; 18(7):1993-2000.

11. Vagetti GC, Oliveira V. Estudo dos níveis de força muscular em idosas e sua adaptação morfofuncional após um período de atividade física. Arq Cien Saúde 2003; 7(1):57-51

12. Kirschke AR, Kocur P, Wilk M, Dylewicz P. The Fullerton Fitness Test as an index of fitness in the elderly. Medical Rehabilitation 2006; 10(2):9-16.

13. Spilker B. Quality of life assessments in clinical trials. New York: Raven Press; 1990.

14. Arnold R, Ranchor AV, Sanderman R, Kempen GI, Ormel J, Suurmeijer TP. The relative contribution of domains of quality of life to overall quality of life for different chronic diseases. Qual Life Res 2004; 13(5):883-896.

15. Pereira RJ, Cotta RMM, Franceschini SCC, Ribeiro RCL, Sampaio RF, Priore SE, Cecon PR. Contribuição dos domínios físico, social, psicológico e ambiental para a QV global de idosos. Revista Psiquiatr Rio Gd Sul 2006; 28(1):27-38.
16. Vagetti GC, Oliveira V, Barbosa Filho VC, Moreira NB, Campos W. Predição da qualidade de vida global em idosas ativas por meio dos domínios do WHOQOL-BREF e do WHOQOL-OLD. Motricidade 2012; 8(Supl. 2):709-718.

17. Ng N, Hakimi M, Byass P, Wilopo S, Wall S. Health and quality of life among older rural people in Purworejo District, Indonesia. Glob Health Action 2010; 3 .

18. Joia LC, Ruiz T. Inquérito populacional sobre o estilo e a qualidade de vida na terceira idade. Rev Bras Med Fam e Com 2006; 2(6):114-130.

19. Lima-costa MF, Barreto S, Giatti L, Uchôa E. Desigualdade social e saúde entre idosos brasileiros: um estudo baseado na Pesquisa Nacional por Amostra de Domicílios. Cad Saude Publica 2003; 19(3):745757.

20. Organização Mundial da Saúde (OMS). Diminuindo diferenças: A prática das políticas sobre determinantes sociais da saúde. Rio de Janeiro: OMS; 2011.

21. Instituto de pesquisa e planejamento urbano de Curitiba (IPPUC). Curitiba em dados. [página na Internet]. [acessado 2012 mar 1]. Disponível em: http://curitibaemdados.ippuc.org.br/Curitiba_ em_dados_Pesquisa.htm

22. Fleck MPA, Louzada S, Xavier M, Chachamovich E. Aplicação da versão em português do instrumento abreviado de avaliação da qualidade de vida "WHOQOL-BREF". Rev Saude Publica 2000; 34(2)178-183.

23. Fleck MPA, Lea OF, Louzada S, Xavier M, Chachamovich E, Vieira G, Santos L, Pinzon V. Desenvolvimento da versão em português do instrumento de Avaliação de qualidade de vida da OMS (WHOQOL-100). Rev Bras Psiquiatr 1999; 21(1):19-28.

24. Chiu MH, Hwang HF, Lee HD, Chien DK, Chen CY, Lin MR. Effect of fracture type on health-related quality of life among older women in Taiwan. Arch Phys Med Rehabil 2012; 93(3);512-519.

25. Associação Brasileira de Empresas de Pesquisa (ABEP). Critério de classificação econômica Brasil. São Paulo: ABEP; 2008.

26. World Health Organization (WHO). Obesity: preventing and managing the global epidemic. Report of a WHO Consultation. Geneva: WHO; 2000.

27. Matsudo S, Araújo T, Matsudo V, Andrade D, Andrade E, Oliveira LC, Braggion G. Questionário Internacional de Atividade Física (IPAQ): estudo de validade e reprodutibilidade no Brasil. Rev Bras Ativ Fís Saúde 2001; 6(2):5-18.

28. World Health Organization (WHO). Global recommendations on physical activity for health. Geneva: WHO; 2010

29. Ekwall A, Lindberg A, Magnusson M. Dizzy - why not take a walk? Low level physical activity improves quality of life among elderly with dizziness. Gerontology 2009; 55(6):652-659.

30. Elavsky S, Mcauley E, Motl RW, Konopack JF. Physical Activity Enhances Long-Term Quality of Life in Older Adults: Efficacy, Esteem, and Affective Influences. Ann Behav Med 2005; 30(2):138-145. 
31. Silva RJS, Smith-Menezes A, Tribess S, Rómo-Perez V, Virtuoso Junior JS. Prevalência e fatores associados à percepção negativa da saúde em pessoas idosas no Brasil. Rev Bras Epidemiol 2012; 15(1):49-62.

32. Lima MG, Barros MB, César CL, Goldbaum M, Carandina L, Ciconelli RM. Health related quality of life among the elderly: a population-based study using SF-36 survey. Cad Saude Publica 2009; 25(10): 2159-2167.

33. Sousa AI, Silver LD. Perfil sociodemográfico e estado de saúde auto-referido entre idosas de uma localidade de baixa renda. Esc Anna Nery Rev Enfermagem 2008; 12(4):706-716.

34. Jakobsson U, Hallberg IR, Westergren A. Overall and health related quality of life among the oldest old in pain. Qual Life Res 2004; 13(1):125-136.

35. Hu J, Wallace DC, Tesh AS. Physical activity, obesity, nutritional health and quality of life in low-income hispanic adults with diabetes. J Community Health Nurs 2010; 27(2):70-83.

36. Alencar NA, Bezerra JCP, Dantas EHM. Evaluation of the levels of physical activity, functional autonomy and quality of life of elderly women members of the family health program. Fit Perf J 2009; 8(5):315-321.

37. American College of Sports Medicine. Exercise and physical activity for older adults. Med Sci Sports Exerc 2009; 41(7):1510-1530.

38. Tamai SAB, Paschoal SMP, Lityoc J, Machado AN, Curiati PK, Prada LF, Jacob-Filho W. Impact of a program to promote health and quality of life of elderly. Inst Ens Pesq Albert Einstein 2011; 9(1):8-13.

39. Mcauley E, Doerksen SE, Morris KS, Motl RW, Hu L, Wójcicki TR, White SM, Rosengren KR. Pathways from physical activity to quality of life in older women. Ann Behav Med 2008; 36(1):13-20.

40. Organização Mundial de Saúde (OMS). Saúde e envelhecimento: Um documento para o debate: Versão Preliminar. Madrid: Instituto de Migração e Serviços Sociais; 2001.

41. Farenzena WP, Argimon IL, Moriguchi E, Portuguez MW. Qualidade de vida em um grupo de idosos de Veranópolis. Rev Kairós 2007; 10(2):225-243.

42. Paskulin L, Vianna L, Molzahn AE. Factors associated with quality of life of Brazilian older adults. Int Nurs Rev 2009; 56(1):109-115.

Artigo apresentado em 19/11/2012

Aprovado em 11/04/2013

Versão final apresentada em 27/04/2013 
